\title{
Saliva molecular testing for SARS-CoV-2: simplifying the diagnosis without losing accuracy
}

\begin{abstract}
Copyright $\odot$ The authors 2021.
\end{abstract} \\ This version is distributed under \\ the terms of the Creative \\ Commons Attribution \\ Non-Commercial Licence 4.0. \\ For commercial reproduction \\ rights and permissions contact \\ permissions@ersnet.org \\ Received: 28 July 2021 \\ Accepted: 22 Sept 2021
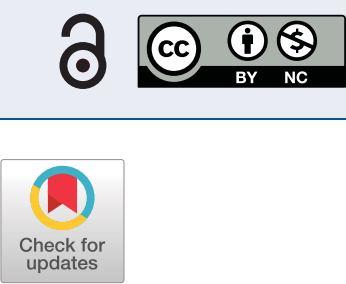

\section{To the Editor:} \\ The possibility to rely on rapid and accurate diagnostic techniques has proved itself crucial during the past year \\ to contain the spread of SARS-CoV-2 infection [1]. Even if quantitative RT-PCR (RT-qPCR) on \\ nasopharyngeal swab (NPS) is still considered the standard for coronavirus disease 2019 (COVID-19) diagnosis, \\ saliva has been evaluated in several studies as a possible alternative to NPS and is currently extensively utilised \\ in South Korea, Germany and Japan [2, 3]. Nonetheless, the use of saliva is still debated, and a rigorous \\ standardisation of the analysis protocol is greatly needed [4-6]. The application of point-of-care technologies on \\ saliva, able to rapidly perform highly specific and sensitive molecular testing, could prove invaluable to allow \\ the diagnosis also in challenging and remote settings by simplifying and speeding up the diagnostic process [1]. \\ To assess the sensitivity and specificity of molecular testing on saliva in comparison to NPS using two \\ different point-of-care platforms (DiaSorin Simplexa: Diasorin, Cypress, CA, USA; and Cepheid Xpert: \\ Cepheid, Sunnyvale, CA, USA), we enrolled a total of 129 individuals into the study. We prospectively \\ collected samples from January 2021 to May 2021, from 21 asymptomatic healthcare workers, taking part \\ in a COVID-19 screening campaign, and from 79 outpatients who had developed mild symptoms \\ consistent with COVID-19 up to 10 days before accessing the preventive medicine unit, the COVID-19 \\ mildly symptomatic outpatients unit or the emergency department of San Raffaele Hospital, Milan. \\ Moreover, we retrieved from the San Raffaele Hospital biobank samples from 29 patients, hospitalised for \\ COVID-19 in March 2020. For each patient, we analysed a self-collected saliva sample and an NPS, \\ collected at the same time by a healthcare worker. This study was approved by the San Raffaele Hospital's \\ ethics committee (protocol number: CLI-PR-2020) and all participants signed informed consent. \\ With the exclusion of the samples collected in March 2020 , that were stored at $-80^{\circ} \mathrm{C}$ immediately after \\ sampling, all samples were preserved at $4^{\circ} \mathrm{C}$ and analysed within $24 \mathrm{~h}$ from collection. DiaSorin Simplexa \\ COVID-19 Direct tests (Simplexa) were performed on saliva diluted 1:1 with saline as per instructions for \\ use, and the same condition was used off label for the Xpert Xpress SARS-CoV-2 kit (Xpert). NPS were \\ analysed with the Xpert Xpress SARS-COV-2 or Roche Cobas SARS-CoV-2 (Cobas) tests, as per \\ manufacturers' instructions. \\ The results obtained on saliva samples collected prospectively in the first months of 2021, demonstrated \\ for both Simplexa and Xpert a specificity of 100\% (95\% CI 93.9-100\%) and a sensitivity of 90.2\% (95\% \\ CI 76.8-97.2\%) when compared to results from NPS. The overall agreement between the two tests \\ performed on saliva was $98 \%$. \\ Since the two kits employed on saliva, as well as those used on NPS, assess different target genes \\ (Simplexa: Orf1ab and S; Xpert: N2 and E; Cobas: E and Orf1ab), we analysed, for the shared targets, the \\ correlation between cycle threshold $(\mathrm{Ct})$ values detected on saliva and on NPS. \\ We identified a positive correlation for Ct values detected on saliva and on NPS for Orf1ab, detected both \\ by Simplexa performed on saliva and by Cobas on NPS (Kendall correlation 0.7704, p<0.0001) as well as \\ for E (Kendall correlation 0.7961, $\mathrm{p}<0.0001$ ) and N2 (Kendall correlation 0.8311, $\mathrm{p}<0.0001$ ), both targets \\ of the Xpert assay performed on saliva and on NPS (figure 1a).

\section{Shareable abstract (@ERSpublications)} \\ This study demonstrated that the use of point of care technologies on saliva represents a valid and \\ highly specific solution to simplify, speed up and broadly distribute the diagnostic process for the \\ control of the COVID-19 epidemic https://bit.ly/3oh4bds \\ Cite this article as: Saluzzo F, Mantegani P, Poletti de Chaurand V, et al. Saliva molecular testing for \\ SARS-CoV-2: simplifying the diagnosis without losing accuracy. Eur Respir J 2021; 58: 2102099 \\ [DOI: 10.1183/13993003.02099-2021].
}


CtE

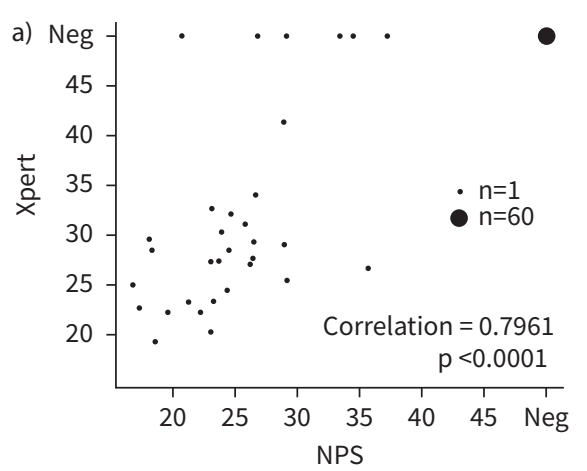

Ct Orf1ab

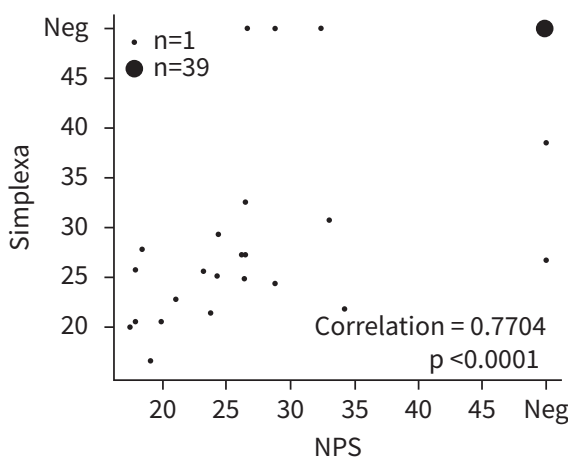

Ct N2

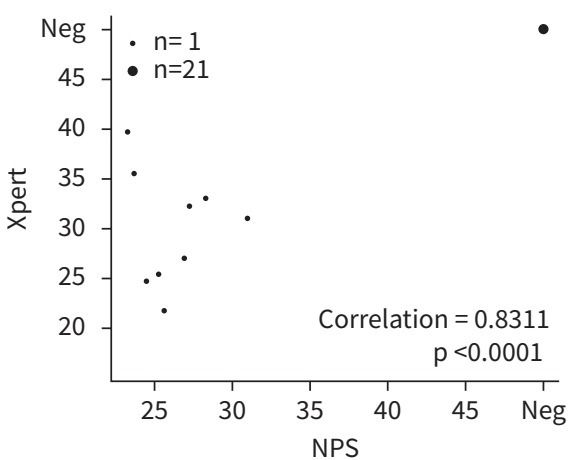

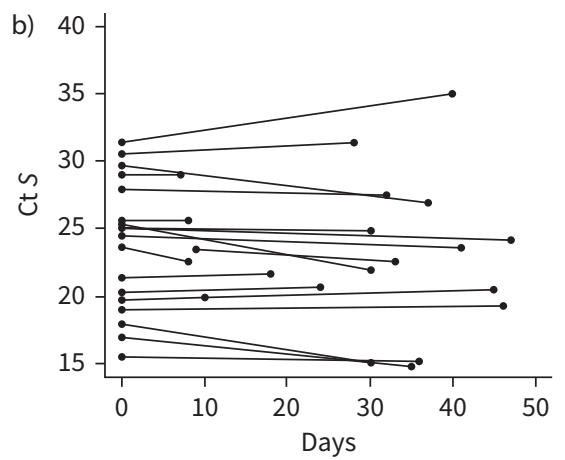
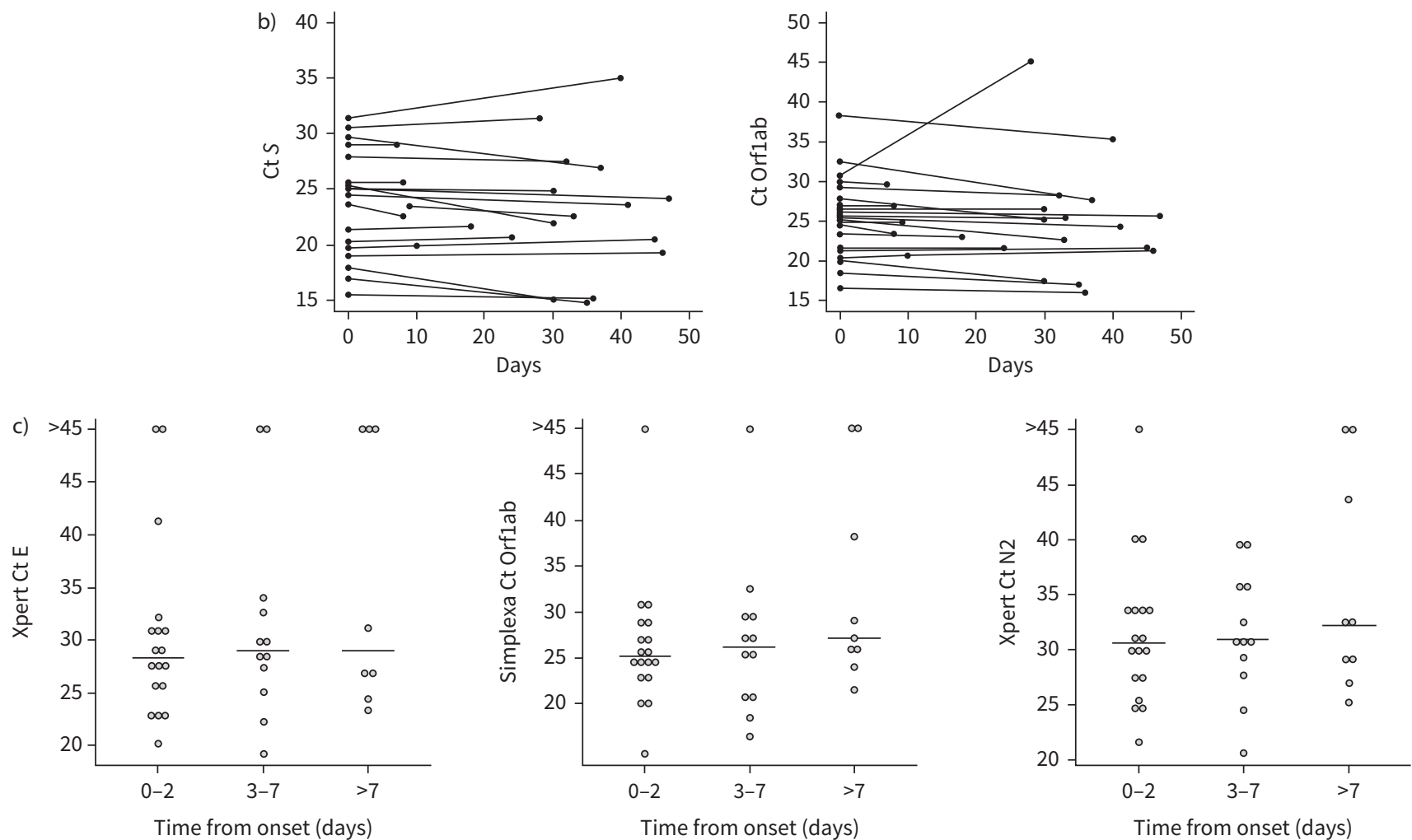

FIGURE 1 a) Cycle threshold (Ct) values comparison between saliva (examined with Simplexa or Xpert) and nasopharyngeal swab (NPS) for different targets (genes $\mathrm{E}$, Orf1ab and N2). b) Ct values before and after storage at $-80^{\circ} \mathrm{C}$ at different timepoints. C) Comparison of $\mathrm{Ct}$ values at different time frames from symptom onset.

Hence, when compared to NPS, both tests on freshly collected saliva appeared to have good sensitivity and specificity as well as a positive correlation for Ct values detected for the shared targets.

We then evaluated the effects of storage at $-80^{\circ} \mathrm{C}$ for up to 45 days on 22 saliva samples that resulted positive for SARS-CoV-2. We analysed the samples with Simplexa before and after the freezing and compared the Ct values for the two different targets (S and Orf1ab). Both S Ct values and Orf1ab Ct values were not significantly different before (median (interquartile range): S Ct 23.8 (20.00-25.50), Orf1ab Ct 24.95 (21.38-27.52)) or after (S Ct 22.6 (20.55-25.4), Orf1ab Ct 24.5 (21.52-26.70)) the freezing (Wilcoxon test 0.0705).

Moreover, the observed difference in Ct values did not appear to be connected to the number of days for which the samples remained stored at $-80^{\circ} \mathrm{C}$, as we did not retrieve a statistically significant correlation between the storage time and the Ct values ( $\mathrm{S}$ : Kendall correlation $-0.0356, \mathrm{p}=0.8206$; Orf1ab: Kendall correlation $-0.0574, \mathrm{p}=0.7128$ ) (figure $1 \mathrm{~b}$ ). 
Nonetheless, once we included in the performance analysis the biobank-collected saliva samples from the COVID-19 inpatients from March 2020 (data not shown), the resulting sensitivity was of 87.14\% (61/70, 95\% CI 76.99-93.95\%) for Simplexa and 91.4\% (64/70, 95\% CI 82.2-96.7\%) for Xpert, and the agreement between Simplexa and Xpert performed on saliva was of 96.1\%.

Considering that the median (interquartile range) time from illness onset to collection of the biobank specimens was 4 days (2-9 days), while for the fresh samples collected in 2021 was 2.5 days (2-4 days), we evaluated whether the time elapsed between symptom onset and sample collection could be a possible factor affecting the Ct values.

When categorising the samples from symptomatic patients in three different categories (0-2 days from symptom onset to collection, 3-7 days and >7 days) for saliva samples, we observed no statistically significant differences between different timeframes in Ct values for either of the targets in analysis (E: Kruskal-Wallis test, $\mathrm{p}=0.80$; Orf1ab: Kruskal-Wallis test, $\mathrm{p}=0.39$; N2: Kruskal-Wallis test, $\mathrm{p}=0.80$ ) (figure $1 \mathrm{c}$ ). Instead, for NPS we observed a statistically significant increase in Ct values for both the E gene (Kruskal-Wallis test, $\mathrm{p}=0.007544$ ) and for the ORF1a/b gene (Kruskal-Wallis test $\mathrm{p}=0.03605$ ).

Both Xpert and Simplexa platforms proved to be practical and easy to use on saliva, and the obtained results demonstrated an overall performance comparable to NPS, with a specificity of $100 \%$ and a sensitivity higher than $90 \%$ for freshly collected samples and higher than $87 \%$ for samples stored at $-80^{\circ} \mathrm{C}$, thus demonstrating the possibility to perform these tests also on frozen samples with only a minimal loss in sensitivity. It is interesting to note that the samples comprised all the different SARS-CoV-2 variants of concern currently represented in Italy (alpha, beta and delta) and both kits' performance was not compromised by such variable.

The tests employed exhibited an overall excellent level of agreement, even when considering the differences identified once we included the biobank samples into the analysis.

As the pandemic evolves, the implementation of a testing strategy based on points of care widespread across the jurisdiction could help to guarantee a prompt on-site diagnosis, allowing the rapid identification and control of clusters and outbreaks, thus protecting the community from disease transmission. Moreover, if this new diagnostic plan would involve the use of highly reliable self-collecting samples directly at patients' homes, such as saliva, we would reduce the burden on healthcare workers, and the costs related to the use of NPS with specific transport medium. This approach would also contribute to drastically decrease the number of possible infectious individuals commuting to the sampling hubs, who could represent a major public health risk.

This diagnostic approach could be easily implemented also in low and middle income countries, where point-of-care platforms are already widely employed for the diagnosis of other illnesses, such as tuberculosis, HIV and viral hepatitis.

In conclusion, our findings support the use of saliva on point-of-care technologies as a valid solution to simplify, speed up and broadly deploy across the territory the diagnostic processes for the control of the COVID-19 epidemic.

\section{Francesca Saluzzo $\oplus^{1}$, Paola Mantegani ${ }^{1}$, Valeria Poletti de Chaurand $\oplus^{1}$, Federica Cugnata ${ }^{2}$, Patrizia Rovere-Querini ${ }^{3}$, Marta Cilla ${ }^{3}$, Patrizia Paola Erba ${ }^{3}$, Sara Racca ${ }^{4}$, Cristina Tresoldi ${ }^{5}$, Caterina Uberti-Foppa $\oplus^{6}$, Clelia Di Serio ${ }^{2}$ and Daniela Maria Cirillo $\oplus^{1}$}

${ }^{1}$ Division of Immunology, Transplantation and Infectious Disease, IRCCS Ospedale San Raffaele, Milan, Italy. ${ }^{2}$ CUSSB-University Center for Statistics in the Biomedical Sciences, Vita-Salute San Raffaele University, Milan, Italy. ${ }^{3}$ IRCCS Ospedale San Raffaele and Vita-Salute San Raffaele University, Milan, Italy. ${ }^{4}$ Laboratorio Microbiologia/Virologia, SMEL, IRCSS Ospedale San Raffaele, Milan, Italy. ${ }^{5}$ Molecular Hematology Unit, IRCCS Ospedale San Raffaele, Milan, Italy. ${ }^{6}$ Division of Infectious Diseases, IRCSS Ospedale San Raffaele and Vita-Salute San Raffaele University, Milan, Italy.

Corresponding author: Daniela Maria Cirillo (cirillo.daniela@hsr.it)

Acknowledgements: We thank DiaSorin Italia and Cepheid Italia for providing the reagents to perform the study free of charge. 
Conflict of interest: F. Saluzzo has nothing to disclose. P. Mantegani has nothing to disclose. V. Poletti de Chaurand has nothing to disclose. F. Cugnata has nothing to disclose. P. Rovere-Querini has nothing to disclose. M. Cilla has nothing to disclose. P.P. Erba has nothing to disclose. S. Racca has nothing to disclose. C. Tresoldi has nothing to disclose. C. Uberti-Foppa has nothing to disclose. C. Di Serio has nothing to disclose. D.M. Cirillo reports provision of tests from Cepheid and DiaSorin during the conduct of the study.

\section{References}

1 Pérez-López B, Mir M. Commercialized diagnostic technologies to combat SARS-CoV2: advantages and disadvantages. Talanta 2021; 225: 121898.

2 Tan SH, Allicock O, Armstrong-Hough M, et al. Saliva as a gold-standard sample for SARS-CoV-2 detection. Lancet Respir Med 2021; 9: 562-564.

3 Wyllie AL, Fournier J, Casanovas-Massana A, et al. Saliva or nasopharyngeal swab specimens for detection of SARS-CoV-2. N Engl J Med 2020; 383: 1283-1286.

4 Kapoor P, Chowdhry A, Kharbanda OP, et al. Exploring salivary diagnostics in COVID-19: a scoping review and research suggestions. BDJ Open 2021; 7: 8.

5 McCormick-Baw C, Morgan K, Gaffney D, et al. Saliva as an alternate specimen source for detection of SARS-CoV-2 in symptomatic patients using cepheid Xpert Xpress SARS-CoV-2. J Clin Microbiol 2020; 58: e01109-20.

6 Wong RC, Wong AH, Ho Yl, et al. Evaluation on testing of deep throat saliva and lower respiratory tract specimens with Xpert Xpress SARS-CoV-2 assay. J Clin Virol 2020; 131: 104593. 